\title{
The clinical characteristics and risk factors for necrotizing pneumonia caused by Mycoplasma pneumoniae in children
}

\author{
Baoying Zheng, Jing Zhao ${ }^{*}$ and Ling Cao*
}

\begin{abstract}
Background: The incidence of necrotizing pneumonia (NP) caused by Mycoplasma pneumoniae (MP) is increasing. We analyzed the clinical characteristics and the risk factors for NP caused by MP.

Methods: A retrospective observational study was conducted in 37 patients with NP caused by MP (NP group) and 74 patients diagnosed with lobar M. pneumoniae pneumonia with no necrosis (control group) who were admitted to our hospital between January 2013 and December 2017. The clinical manifestations, laboratory data, imaging findings, treatments and outcomes were analyzed.

Results: The proportion of females, the incidence of pleural effusion, fever duration, hospitalization days, white blood cell count, neutrophil ratio, D-dimer level and use of other types of antibiotics were higher in the NP group than in the control group $(P<0.05)$. The control group exhibited a greater use of low molecular weight heparin $(\mathrm{LMWH})$ than the NP group $(P<0.05)$. According to the multivariate logistic regression analysis, a white blood cell count $>12.3 \times 10^{9} / \mathrm{L}$ (Odds ratio, $\left.\mathrm{OR}=6.412\right)$, a neutrophil ratio $>73.9 \%(\mathrm{OR}=6.081)$ and $\mathrm{D}$-dimer level $>1367.5 \mathrm{ng} /$ $\mathrm{mL}(\mathrm{OR}=8.501)$ were risk factors for pulmonary necrosis caused by MP. Furthermore, the use of $L M W H(O R=0.074)$ reduced the risk of pulmonary necrosis.

Conclusions: NP is a rare complication of severe Mycoplasma pneumoniae pneumonia (SMPP), and although the clinical course is longer than common MP infection, the necrotic area is absorbed gradually. In patients with SMPP presenting with lobar consolidation, a white blood cell count $>12.3 \times 10^{9} / \mathrm{L}$, a neutrophil ratio $>73.9 \%$ and D-dimer level $>1367.5 \mathrm{ng} / \mathrm{mL}$ are risk factors for pulmonary necrosis, and the use of LMWH reduces the risk of pulmonary necrosis.
\end{abstract}

Keywords: Necrotizing pneumonia, Mycoplasma pneumoniae, Children, Risk factor

\section{Background}

Mycoplasma pneumoniae (MP) is one of the most important pathogens responsible for community-acquired pneumonia in school-aged children and young adults [1]. The clinical course of $M$. pneumoniae pneumonia (MPP) is typically mild and self-limiting [2], but severe Mycoplasma pneumoniae pneumonia (SMPP) has been

\footnotetext{
*Correspondence: Janezhao0607@163.com; caoling9919@163.com Department of Pulmonology, Capital Institute of Pediatrics-Peking University Teaching Hospital, Beijing, China
}

reported, and a number of severe pulmonary complications, including obliterative bronchitis, bronchiectasis and necrotizing pneumonia (NP), have been reported [3].

NP is a rare manifestation of MP infection [4], and the literature focused on NP caused by M. pneumoniae infection is scarce; no study has analyzed the risk factors for pulmonary necrosis. In the present study, we reviewed the records of 37 patients with NP caused by MP who were admitted to our hospital and compared them with 74 patients who were diagnosed with lobar

(c) The Author(s). 2020 Open Access This article is licensed under a Creative Commons Attribution 4.0 International License, which permits use, sharing, adaptation, distribution and reproduction in any medium or format, as long as you give appropriate credit to the original author(s) and the source, provide a link to the Creative Commons licence, and indicate if changes were made. The images or other third party material in this article are included in the article's Creative Commons licence, unless indicated otherwise in a credit line to the material. If material is not included in the article's Creative Commons licence and your intended use is not permitted by statutory regulation or exceeds the permitted use, you will need to obtain permission directly from the copyright holder. To view a copy of this licence, visit http://creativecommons.org/licenses/by/4.0/ The Creative Commons Public Domain Dedication waiver (http://creativecommons.org/publicdomain/zero/1.0/) applies to the data made available in this article, unless otherwise stated in a credit line to the data. 
MPP without necrosis. We analyzed the clinical characteristics of these patients and the risk factors for NP caused by MP.

\section{Methods}

\section{Patients}

Thirty-seven patients with NP caused by MP (NP group) who were admitted to The Affiliated Children's Hospital of Capital Institute of Pediatrics from January 1, 2013, to December 31, 2017, were identified. Seventy-four patients diagnosed with lobar MPP without necrosis (control group) were also included. This study was approved by the Ethics Committee of Capital Institute of Pediatrics-Peking University Teaching Hospital (no. SHERLL2019061).

\section{Diagnostic criteria}

A diagnosis of pneumonia was defined as follows [5]: acute respiratory infection symptoms (fever, cough or wheezing) upon a physical examination and chest imaging with infiltrates. Severe pneumonia was defined as pneumonia plus one of the following characteristics [6]: (1) a poor general condition, (2) an increased respiratory rate (infants $>70$ breaths $/$ min and older children $>50$ breaths/min), (3) dyspnea and cyanosis, (4) multilobe involvement or involvement of $\geq 2 / 3$ of the lung, (5) extrapulmonary complications, (6) pleural effusion, and (7) transcutaneous oxygen saturation in room air $\leq 92 \%$.

The MP infection was confirmed by conducting a serological test (MP-IgM-positive and an antibody titer $\geq 1: 160$ or a four-fold or greater increase in the titer) and MP nucleic acid detection from the nasopharyngeal aspirate and BAL and/or pleural effusion [7, 8]. In our study, the antibody titers were $\geq 1: 640$ in both the NP group and control group. In addition, 55 (49.5\%) patients also tested for nucleic acids of M. pneumoniae in nasopharyngeal or bronchoalveolar lavage fluid, and the results were positive. SMPP was defined as severe pneumonia with an MP infection.

The diagnosis of NP mainly depends on chest imaging. In plain chest radiographs, necrotic lesions may present as density-reduced areas and cavity lesions. Chest computed tomography (CT) manifestations of NP were the destruction of normal lung parenchymal structures and a decrease in parenchymal enhancement with structures that were gradually replaced by multiple small air- or fluid-filled cavities with thin, nonenhanced walls [9-11].

\section{Inclusion and exclusion criteria}

Because all patients in the NP group and control group were diagnosed with SMPP, a chest CT was performed on patients in both groups while they were in the hospital. Therefore, the diagnosis of NP was based on the CT manifestations. Forty-three (38.7\%) patients underwent a contrast-enhanced chest CT.
The NP group included patients who were diagnosed with community-acquired pneumonia caused by MP and in whom necrotic lesions were observed on the chest CT. All patients presented with lobar consolidation early in the course of the disease. Cultures for bacteria and fungus were negative in all patients. Bacterial nucleic acids were not detected.

For the control group, we selected children diagnosed with SMPP with lobar consolidation and in whom the lung lesions gradually improved. No necrotic lesions were observed within 3 months. Cultures for bacteria and fungus were negative. Bacterial nucleic acids were not detected. One hundred eighty-four patients met these conditions to match the NP group and had complete clinical data available. The patients were numbered, and 74 patients were randomly selected using the random number Table. A statistically significant difference in age was not observed between the two groups $(P>0.05)$.

Children who presented an immunocompromised state or with chronic lung diseases were excluded from this study. We also excluded children with other cavitary diseases, such as lung abscess, pulmonary cyst with infection, and pulmonary tuberculosis. In addition, patients with a family history of embolism were excluded.

\section{Data collection}

Clinical information was retrospectively collected from the medical records of the patients. The age, sex, clinical symptoms and signs, intrapulmonary and extrapulmonary complications, fever duration, hospitalization days and treatments were recorded. The maximum levels of the laboratory tests observed during hospitalization, including white blood cell (WBC) counts, neutrophil counts, and levels of C-reactive protein (CRP), procalcitonin (PCT), lactate dehydrogenase (LDH), and D-dimer, were recorded. Blood, pleural effusion and nasopharyngeal aspirate/bronchoalveolar lavage fluid cultures, virus antigen detection assays (respiratory syncytial viruses, adenovirus, metapneumovirus, influenza, and parainfluenza), interferon- $\gamma$ release assays and $\mathrm{T}$ cell spot tests (T-SPOTs) for a tuberculosis infection were performed. Thirty-five (31.5\%) patients underwent bacterial nucleic acid detection (Acinetobacter baumannii, Escherichia coli, Klebsiella pneumoniae, Streptococcus pneumoniae, Chlamydia pneumoniae, Staphylococcus aureus, Haemophilus influenzae, methicillin-resistant Staphylococcus, Legionella pneumophila, Pseudomonas aeruginosa, Pseudomonas maltophilia, Mycobacterium tuberculosis). Fifty-five (49.5\%) patients were tested for nucleic acids of $M$. pneumoniae. A chest CT was performed during hospitalization. Repeated imaging examinations, including plain chest radiographs and chest CT, were performed within the followup period. Some children underwent fiber bronchoscopy 
with bronchoalveolar lavage. An electrocardiogram, echocardiography and abdominal ultrasound were performed in all patients.

\section{Statistical analysis}

The statistical analyses were performed using SPSS software (IBM, version 22). Normally distributed data are reported as means \pm standard deviations (SDs). Data with a skewed distribution are presented as median values (interquartile ranges: Q1, Q3). The different groups were compared using a two-sample $t$ test or the Mann-Whitney $U$ test. The statistical significance of the differences in the categorical variables was determined using the chi-square test and Fisher's exact test. Statistical significance was determined at the two-tailed 0.05 level. A multivariate logistic regression analysis was performed to identify the risk factors for NP caused by MP. Some continuous variables, such as the fever duration, WBC counts, neutrophil ratio, CRP levels and D-dimer levels, were categorized into the 30th percentile, 60th percentile and 90th percentile.

\section{Results}

\section{Clinical features (Tables 1 and 2)}

All patients presented with a fever and cough. The total fever duration was $18.3 \pm 8.7$ days in NP group and $11.4 \pm 2.9$ days in the control group. Three patients (8.1\%) in the NP group suffered from chest pain, and none of the patients in the control group presented with chest pain. Four patients (10.8\%) in the NP group and 3 patients $(4.1 \%)$ in the control group suffered from dyspnea. Twenty-three patients $(62.2 \%)$ in the NP group and 29 patients (39.2\%) in the control group had pleural effusion. Three patients $(8.1 \%)$ in the NP group and 9 patients (12.2\%) in the control group experienced atelectasis. Sixteen patients (43.2\%) in the NP group and 38 patients (51.4\%) in the control group presented with extrapulmonary manifestations, of which abnormal liver function was the most common symptom.

Significant differences in age, the incidence of atelectasis and extrapulmonary complications were not observed between the two groups $(P>0.05)$. The NP group included a higher proportion of females than the control group $(P<0.05)$. The total fever duration and the incidence of chest pain and pleural effusion in the NP group were higher than in the control group $(P<0.05)$.

\section{Laboratory data}

A portion of the laboratory data are presented in Table 3. A significant difference in LDH levels was not observed between the two groups $(P>0.05)$. Higher CRP levels, WBC counts, neutrophil ratios, and D-dimer levels were observed in the NP group than in the control group $(P<0.05)$.
Table 1 Clinical information and treatment of the two groups

\begin{tabular}{llll}
\hline Clinical information and treatment & $\begin{array}{l}\text { NP group } \\
(n=37)\end{array}$ & $\begin{array}{l}\text { Control } \\
(n=74)\end{array}$ & \\
\hline Clinical presentation ( $\mathrm{n}, \%)$ & & & \\
Fever & $37,100 \%$ & $74,100 \%$ & - \\
Cough & $37,100 \%$ & $74,100 \%$ & - \\
Chest pain & $3,8.1 \%$ & 0 & $0.035^{\mathrm{a}}$ \\
dyspnea & $4,10.8 \%$ & $3,4.1 \%$ & 0.334 \\
Extrapulmonary complications (n, \%) & & & \\
Elevated liver enzyme level & $10,27 \%$ & $28,37.8 \%$ & 0.258 \\
Electrolyte disturbance & $4,10.8 \%$ & $6,8.1 \%$ & 0.907 \\
Hypoproteinemia & $4,10.8 \%$ & $2,2.7 \%$ & 0.182 \\
Coronary dilatation & 0 & $1,1.4 \%$ & $1.000^{\mathrm{a}}$ \\
Pericardial effusion & 0 & $1,1.4 \%$ & $1.000^{\mathrm{a}}$ \\
Polymorphic erythema & 0 & $1,1.4 \%$ & $1.000^{\mathrm{a}}$ \\
Treatment (n, \%) & & & \\
Administered other antibiotics & $34,91.9 \%$ & $51,68.9 \%$ & 0.007 \\
Noninvasive ventilation & $1,2.7 \%$ & 0 & 0.722 \\
Low molecular weight heparin & $13,35.1 \%$ & $42,58.1 \%$ & 0.032 \\
Methylprednisolone & $30,81.1 \%$ & $65,87.8 \%$ & 0.339 \\
Intravenous immunoglobulin & $10,27 \%$ & $8,10.8 \%$ & 0.029 \\
Fiber bronchoscope examination & $23,62.2 \%$ & $48,64.9 \%$ & 0.780 \\
Mucous congestion, edema & $23,100 \%$ & $48,100 \%$ & 0.780 \\
Bronchitis obliterans & $1,4.3 \%$ & 0 & $0.324^{\mathrm{a}}$ \\
Plastic bronchitis & $1,4.3 \%$ & 0 & $0.324^{\mathrm{a}}$ \\
\hline
\end{tabular}

${ }^{\text {a }}$ Fisher's exact test

In the NP group, PCT levels were measured in 32 patients; 25 (78.1\%) patients presented normal PCT levels $(<0.5 \mathrm{ng} / \mathrm{mL})$, but only $2(6.3 \%)$ presented PCT levels $>$ $10 \mathrm{ng} / \mathrm{mL}$. In the control group, PCT levels were measured in 70 patients; 58 (82.9\%) presented normal PCT levels and no patient presented a PCT level $>10 \mathrm{ng} / \mathrm{mL}$. A statistically significant difference in the proportion of patients with increased PCT levels was not observed between the two groups $(P>0.05)$.

Table 2 Comparison of the clinical indexes

\begin{tabular}{llll}
\hline Clinical index & $\begin{array}{l}\text { NP group } \\
(n=37)\end{array}$ & $\begin{array}{l}\text { Control group } \\
(n=74)\end{array}$ & $P$ value \\
\hline Female (n, \%) & $28,75.7 \%$ & $41,55.4 \%$ & 0.038 \\
Age (years) & $6.7 \pm 2.5$ & $7.1 \pm 2.3$ & 0.402 \\
Total fever duration (days) & $18.3 \pm 8.7$ & $11.4 \pm 2.9$ & $<0.001$ \\
Pleural effusion (n, \%) & $23,62.2 \%$ & $29,39.2 \%$ & 0.027 \\
Atelectasis (n, \%) & $3,8.1 \%$ & $9,12.2 \%$ & 0.748 \\
Extrapulmonary & $16,43.2 \%$ & $38,51.4 \%$ & 0.197 \\
Complications (n, \%) & & & \\
\hline
\end{tabular}

Data are presented as means \pm SDs 
Table 3 Comparisons of laboratory data

\begin{tabular}{llll}
\hline Laboratory data & NP group $(n=37)$ & $\begin{array}{l}\text { control group } \\
(n=74)\end{array}$ & $P$ value \\
\hline WBC count $\left(\times 10^{9} / \mathrm{L}\right)$ & $13.28(9.74,15.92)$ & $10.05(8.4,11.98)$ & $<0.001$ \\
Neutrophil $(\%)$ & $74.87 \pm 8.34$ & $67.46 \pm 9.11$ & $<0.001$ \\
CRP $(\mathrm{mg} / \mathrm{L})$ & $62.7(32.9,170)$ & $38.67(20.98,85.6)$ & 0.010 \\
LDH $(\mathrm{U} / \mathrm{L})$ & $524(329,616)$ & $436.5(341.75,522.5)$ & 0.215 \\
D-dimer $(\mathrm{ng} / \mathrm{mL})$ & $1411(930.9,3363.5)$ & $859(469.5,1333.75)$ & $<0.001$ \\
\hline
\end{tabular}

Data are presented as means \pm SDs or medians $(\mathrm{Q} 1, \mathrm{Q} 3)$

In the NP group, two patients were coinfected with respiratory syncytial viruses, and one patient was coinfected with adenovirus. In the control group, one patient was coinfected with adenovirus, and one was coinfected with parainfluenza virus.

\section{Radiological findings}

In the NP group, chest CT showed lung consolidation in the early stage of the disease; 32 patients presented unilateral lung consolidation, with one or more affected lobes. Decreased enhancement areas were observed in 17 patients on the contrast-enhanced chest CT images, and pulmonary cavities were observed in 22 patients. The mean time from the onset of symptoms to the discovery of necrotic lesions was $24.92 \pm 10.84$ days. Changes in CT signs were recorded in 12 patients during follow-up. Eight patients underwent a chest CT examination at 2-3 months after the onset of disease. Pulmonary consolidation was observed in 4 patients. One patient presented cavities in the lung, 1 presented with atelectasis and 1 presented with pleural thickness. A chest CT was performed after 6 months in 3 patients, with 1 presenting cavities in the lung. Two patients underwent a chest $\mathrm{CT}$ examination after 1 year, one had atelectasis and bronchiectasis, and one had fibrous stripes in the lung.

\section{Treatments and outcomes}

Because quinolones are not approved for use in children under 18 and tetracycline is not approved for use in children under 8 by the China Food and Drug Administration (CFDA), we treated the MP infection with macrolides. Many patients, even up to $91.9 \%$ in the NP group, were administered other antibiotics in addition to macrolides, such as a cephalosporin, vancomycin, or imipenem, because of the severity of the disease and the significantly increased WBC counts and CRP and PCT levels. Methylprednisolone (1-2 mg/kg, 2-3 times a day) and intravenous immunoglobulin were administered to some patients with an acute onset, rapid progression and serious illness, namely, 81.8 and $27 \%$ in the NP group and 87.8 and $10.8 \%$ in the control group, respectively. In out hospital, low molecular weight heparin (LMWH) was administered at a dose of $50-100 \mathrm{mg} / \mathrm{kg}, 1-2$ times a day when Ddimer levels increased. In the present study, some children had been treated in other hospitals for a period of time before arriving at our hospital, and LMWH was not applied before the necrotic lesion appeared, even if the D-dimer levels increased. Thirteen patients (35.1\%) in the NP group and 42 patients $(58.1 \%)$ in the control group were treated with LMWH (Table 1).

Significant differences in the use of LMWH and other antibiotics were observed. The use of LMWH in the control group was greater than in the NP group $(P<$ 0.05 ), and the use of other antibiotics and intravenous immunoglobulin in the NP group was greater than in the control group $(P<0.05)$. The median number of hospitalization days was 22 days in the NP group and 11 days in the control group. The NP group was hospitalized for more days than the control group $(P<0.05)$.

\section{Multivariate logistic regression analysis}

According to the multivariate logistic regression analysis (Table 4), a WBC count $>12.3 \times 10^{9} / \mathrm{L}(\mathrm{OR}=6.412)$, a neutrophil ratio $>73.9 \% \quad(\mathrm{OR}=6.081)$ and $\mathrm{D}$-dimer level $>1367 \mathrm{ng} / \mathrm{mL} \quad(\mathrm{OR}=8.501)$ were risk factors for pulmonary necrosis caused by MP. Furthermore, the use of LMWH $(\mathrm{OR}=0.074)$ reduced the risk of pulmonary necrosis.

Table 4 Results of the multivariate logistic regression analysis

\begin{tabular}{llll}
\hline Parameter & OR & $95 \% \mathrm{Cl}$ & $P$ value \\
\hline Fever duration $(<10$ days $)$ & 1 & & \\
10-14 days & 0.559 & $0.115-2.725$ & 0.472 \\
$>14$ days & 4.719 & $0.943-23.608$ & 0.059 \\
WBC count $\left(<9.5 \times 10^{9} / \mathrm{L}\right)$ & 1 & & \\
$9.5 \times 10^{9}-12.3 \times 10^{9} / \mathrm{L}$ & 1.886 & $0.42-8.476$ & 0.408 \\
$>12.3 \times 10^{9} / \mathrm{L}$ & 6.412 & $1.483-27.728$ & 0.013 \\
Neutrophil ratio $(<66.8 \%)$ & 1 & & \\
66.8-73.9\% & 1.751 & $0.395-7.767$ & 0.461 \\
$>73.9 \%$ & 6.081 & $1.149-32.19$ & 0.034 \\
CRP $(<32 \mathrm{mg} / \mathrm{L})$ & 1 & & \\
32-82 mg/L & 0.777 & $0.158-3.819$ & 0.757 \\
$>32 \mathrm{mg} / \mathrm{L}$ & 0.956 & $0.158-5.781$ & 0.961 \\
D-dimer $(<635.8 \mathrm{ng} / \mathrm{mL})$ & 1 & & \\
635.8-1367.5 ng/mL & 1.999 & $0.158-5.781$ & 0.388 \\
$>1367.5 \mathrm{ng} / \mathrm{mL}$ & 8.501 & $1.282-56.385$ & 0.027 \\
Female & 3.734 & $0.94-14.836$ & 0.061 \\
Pleural effusion & 2.632 & $0.82-8.45$ & 0.104 \\
Use of LMWH & 0.074 & $0.015-0.36$ & 0.001 \\
\hline
\end{tabular}




\section{Discussion}

MP is considered an important pathogen that causes community-acquired pneumonia in children [3, 12]. In China, SMPP is becoming increasingly common, and is characterized by a rapid onset, rapid progression, severe pulmonary lesions, and prolonged course, with a persistent high fever and severe cough as the clinical symptoms. Intrapulmonary and extrapulmonary complications are often associated with SMPP. A number of severe pulmonary complications, including NP, have been reported.

NP is characterized by the necrosis and liquification of the lung tissues, which are replaced by cavities that are surrounded by walls of varying thicknesses $[3,13]$. If not adequately treated, NP may lead to complications, including bronchopleural fistula, empyema, respiratory failure, and septic shock [14]. Bacterial infections, particularly infections of Streptococcus pneumoniae and Staphylococcus aureus, are the most common etiology $[13,15]$. Since NP caused by MP infection was first reported by Oermann [16] in 1997, an increasing number of cases have been reported in the literature.

When the lungs are necrotic, a child often has no complaints of special discomfort. Lung necrosis can be detected at an early stage through contrast-enhanced chest $\mathrm{CT}$, but it is undetectable in plain chest radiographs. Chest CT shows lung consolidation in the early stage of the disease, and decreased parenchymal enhancement is observed on contrast-enhanced chest CT. Finally, pulmonary cavities are observed. In the present study, the mean time from the onset of symptoms to the discovery of necrotic lesions was $24.92 \pm 10.84$ days. Previous studies $[4,9,10,17]$ have showed that the consolidation and necrotic area will be absorbed gradually, and the lung cavity disappears. Residual fibrous stripes, atelectasis, and lobar cystic degeneration have been observed in some patients within 3-6 months; furthermore, these signs may persist for $1-3.5$ years. In our study, most of the patients lived in other provinces, and longterm follow-up was difficult to achieve. Follow-up showed the disappearance of most of the cavity lesions within 3-6 months, consistent with previous reports.

In patients with SMPP, local hypoxia, ischemia and acidosis occur. In addition, the direct invasion of pathogens and their toxins will activate the coagulation system and form a hypercoagulable state. At the same time, the fibrinolysis, kinin and complement systems in the body will also be activated successively, which may cause the abnormal coagulation function [18]. Thromboembolism due to MP infection has been reported in the literature [19]. As a specific marker of fibrinolysis, D-dimer reflects the capacity to dissolve fibrin, and increasing evidence suggests that coagulation function is closely related to the inflammatory response [20]. The clinical symptoms and the chest imaging findings of patients with MPP presenting with elevated D-dimer levels are more serious than patients with normal D-dimer levels $[21,22]$. In our study, the NP group presented a noticeably higher D-dimer level than the control group, consistent with other reports [23]. Furthermore, a D-dimer level $>1367 \mathrm{ng} / \mathrm{mL} \quad(\mathrm{OR}=8.501)$ was a risk factor for pulmonary necrosis. Therefore, when D-dimer levels increase significantly, we should pay attention to the possibility of pulmonary necrosis.

Currently, LMWH is consistently administered as a treatment for hypercoagulation. In addition to anticoagulant and antithrombotic functions, LMWH has many biological activities and pharmacological mechanisms, such as anti-inflammatory effects, anti-fibrosis activity, immune regulation and cell proliferation inhibition $[24,25]$. As shown in the study by Zhang CF et al. [22], early use of LMWH accelerates the absorption of lung lesions, shortens the hospitalization time and improves the prognosis of children with SMPP. In our study, the multivariate logistic regression analysis showed that use of LMWH $(\mathrm{OR}=0.074)$ reduced the risk of pulmonary necrosis, indicating that treatment with LMWH played an important role in SMPP.

According to the multivariate logistic regression analysis, a WBC count $>12.3 \times 10^{9} / \mathrm{L}$ and a neutrophil ratio $>73.9 \%$ were also risk factors for pulmonary necrosis. However, WBC counts and neutrophil ratios are easily affected by other factors, such as coinfection with bacteria and the use of glucocorticoids. Therefore, their predictive effects must be analyzed together with other indicators.

The present study had several limitations. First, it was a retrospective observational study, and the patients enrolled were from a single center. Second, the sample size of the NP group was relatively small.

\section{Conclusions}

In conclusion, NP is a rare complication of SMPP, and although the clinical course is longer than a common MP infection, the necrotic area is absorbed gradually. In patients with SMPP presenting with lobar consolidation, a $\mathrm{WBC}$ count $>12.3 \times 10^{9} / \mathrm{L}$, a neutrophil ratio $>73.9 \%$ and D-dimer level $>1367.5 \mathrm{ng} / \mathrm{mL}$, we should be alert to the occurrence of pulmonary necrosis, and the use of LMWH reduced the risk of pulmonary necrosis.

\section{Abbreviations \\ CFDA: China Food and Drug Administration; CRP: C-reactive protein; $C T$ : computed tomography; LDH: lactate dehydrogenase; LMWH: low molecular weight heparin; MP: Mycoplasma pneumoniae; NP: necrotizing pneumonia; PCT: procalcitonin; SDs: standard deviations; SMPP: severe Mycoplasma pneumoniae pneumonia; T-SPOTs: T cell spot tests; WBC: white blood cell}

\section{Acknowledgements}

We thank Dr. Jun-ting Liu for his assistance with the statistical analysis of the data. 


\section{Authors' contributions}

All authors contributed to the study conception and design. BYZ analyzed the data and drafted the manuscript, LC and JZ revised it. All authors read and approved the final manuscript.

\section{Funding}

This study was supported by the Beijing Municipal Science \& Technology Commission through grant no. Z171100001017081.The funders had no role in study plan, design, data collection and analysis, interpretation of the results, decision to publish, or preparation of the manuscript.

\section{Availability of data and materials}

Datasets analyzed in this study can be obtained from the corresponding author upon reasonable request.

\section{Ethics approval and consent to participate}

This study was approved by the Ethics Committee of Capital Institute of Pediatrics-Peking University Teaching Hospital (no. SHERLL2019061). All data collected were stripped of patient identifiers, with no possibility to track the data to an individual person's identity. The data in the study were obtained with the permission of the Capital Institute of Pediatrics-Peking University Teaching Hospital.

\section{Consent for publication}

Not applicable.

\section{Competing interests}

The authors declare that they have no competing interests.

Received: 1 February 2020 Accepted: 20 May 2020

Published online: 01 June 2020

\section{References}

1. Okada T, Morozumi M, Tajima T, et al. Rapid effectiveness of minocycline or doxycycline against macrolide-resistant mycoplasma pneumoniae infection in a 2011 outbreak among Japanese children. Clin Infect Dis. 2012;55(12): 1642-9.

2. Chiu CY, Chiang LM, Chen TP. Mycoplasma pneumoniae infection complicated by necrotizing pneumonitis with massive pleural effusion. Eur J Pediatr. 2006;165(4):275-7.

3. Wang Y, Xu D, Li S, et al. Mycoplasma pneumoniae-associated necrotizing pneumonitis in children. Pediatr Int. 2012;54(2):293-7.

4. Wang RS, Wang SY, Hsieh KS, et al. Necrotizing pneumonitis caused by mycoplasma pneumoniae in pediatric patients:report of five cases and review of literature. Pediatr Infect Dis J. 2004;23(6):564-7.

5. Shen KL, Jiang ZF. Pneumonia. In: Hu YM, Jiang ZF, editors. Zhu Futang's practical pediatrics. 7th ed. Bei Jing: People's Medical Publishing House; 2002. p. $1175-80$.

6. Subspecialty Group of Respiratory Diseases, The Society of Pediatrics, Chinese Medical Association, Editorial Board, Chinese Journal of Pediatrics. Guidelines for management of community acquired pneumonia in children (the revised edition of 2013) (I). Zhonghua Er Ke Za Zhi. 2013;51(10):745-52.

7. Respiratory Branch of Chinese Pediatric Society of Editorial Board of Chinese Journal of Applied Clinical Pediatrics. Expert consensus on diagnosis and treatment of mycoplasma pneumoniae pneumonia in children (2015). Chin J Appl Clin Pediatr. 2015;30(17):1304-8.

8. Lee WJ, Huang EY, Tsai CM. Role of Serum Mycoplasma pneumoniae $\lg \mathrm{A}, \lg \mathrm{M}$, and $\lg \mathrm{G}$ in the diagnosis of Mycoplasma pneumoniae-related pneumonia in school-age children and adolescents. Clin Vaccine Immunol. 2017;24(1):e 00471-16.

9. Wang $X$, Zhong $L$, Chen ZM. Necrotizing pneumonia caused by refractory mycoplasma pneumonia pneumonia in children. World J Pediatr. 2018;14(4): 344-9.

10. Li SR, Mu JH, Chang L, et al. Chest CT features and outcome of necrotizing pneumonia caused by mycoplasma pneumoniae in children (report of 30 cases). Zhonghua Er Ke Za Zhi. 2013;51(3):211-5.

11. Donnelly LF, Klosterman LA. Cavitary necrosis complicating pneumonia in children:sequetial finding on chest radiography. AJR Am J Roentgenol. 1998; 171(1):253-6.
12. San Martin I, Zarikian SE, Herranz M, et al. Necrotizing pneumonia due to mycoplasma in children:an uncommon presentation of a common disease. Adv Respir Med. 2018;86(6):305-9.

13. Spencer DA, Thomas MF. Necrotising pneumonia in children. Paediatr Respir Rev. 2014;15(3):240-5.

14. Ramgopal S, Ivan Y, Medsinge A, et al. Pediatric necrotizing pneumonia: a case report and review of the literature. Pediatr Emerg Care. 2017;33(2):112-5.

15. Shang $Y X$, Feng Y. Necrotizing pneumonia in children. J Clin Pediatr. 2013; 31(8):701-5.

16. Oermann C, Sockrider MM, Langston C. Severe necrotizing pneumonitis in a child with mycoplasma pneumoniae infection. Pediatr Pulmonol. 1997;24(1): $61-5$.

17. Song L, Peng Y, Liu ZM, et al. Imaging features of necrotic mycoplasma pneumonia in children. Chin J Med Imaging Technol. 2012;28(3):397-400.

18. Nastasijević Borovac D, Petković TR, Pejčić T, et al. Role of D-dimer in predicting mortality in patients with community-acquired pneumonia. Med Glas (Zenica). 2014;11(1):37-43.

19. Zhou YP, Zhuang YP. Changes of coagulation function and its clinical significance in children with mycoplasma pneumonia. Chin J Clin. 2016; 10(19):2962-4.

20. Fan $\mathrm{M}$, Chen $\mathrm{CH}$, Qi YZ. Analysis of the relationship between plasma levels of D-dimer, CRP and the severity of mycoplasma pneumoniae pneumonia. Clin Res. 2017;25(5):7-8,14.

21. Tan SS, Cao L. Therapeutic effect of low molecular weight heparin on adjuvant treatment of mycoplasma pneumoniae pneumonia with elevated D-dimer in children. Chin J Appl Clin Pediatr. 2018;33(16):1242-5.

22. Zhang $C F, H e ~ Y X, W u R H$, et al. Efficacy of low molecular weight heparin in the treatment of severe mycoplasma pneumoniae pneumonia in children with elevated D-dimer. Beijing Med J. 2019;41(3):183-6.

23. Wang RZ, Xi YL, Guo B, et al. Imaging characteristics and serum C-reactive protein, plasma D-dimer change of necrotizing pneumonia caused by mycoplasma pneumoniae infection in children. J Pract Radiol. 2019;35(6): 952-5.

24. Fan Y, Jiang M, Gong D, et al. Efficacy and safety of low-molecular-weight heparin in patients with sepsis: a meta-analysis of randomized controlled trials. Sci Rep. 2016;6:25984.

25. Han YC. Pharmacology and clinical application of low molecular weight heparin. Guide China Med. 2016;14(34):291-2.

\section{Publisher's Note}

Springer Nature remains neutral with regard to jurisdictional claims in published maps and institutional affiliations.

\section{Ready to submit your research? Choose BMC and benefit from:}

- fast, convenient online submission

- thorough peer review by experienced researchers in your field

- rapid publication on acceptance

- support for research data, including large and complex data types

- gold Open Access which fosters wider collaboration and increased citations

- maximum visibility for your research: over $100 \mathrm{M}$ website views per year

At BMC, research is always in progress.

Learn more biomedcentral.com/submissions 